\title{
Ultrasound assessment of fetal cardiac function
}

\section{Fàtima Crispi}

MD, PhD

\section{Brenda Valenzuela- \\ Alcaraz \\ MD}

\section{Monica Cruz-Lemini $\mathrm{MD}, \mathrm{PhD}$}

\section{Eduard Gratacós}

$\mathrm{MD}, \mathrm{PhD}$

Fetal and Perinatal

Medicine Research Group

Institut d'Investigacions

Biomèdiques August Pi i

Sunyer

University of Barcelona

Barcelona

Spain

Department of Maternal-

Fetal Medicine

Institut Clínic de Gineco-

logia

Obstetrícia i Neonatologia

Hospital Clinic

Barcelona

Spain

Centro de Investigación

Biomédica en Red en

Enfermedades Raras

Barcelona

Spain

Correspondence to email gratacos@clinic.ub.es

\begin{abstract}
Introduction: Fetal heart evaluation with US is feasible and reproducible, although challenging due to the smallness of the heart, the high heart rate and limited access to the fetus. However, some cardiac parameters have already shown a strong correlation with outcomes and may soon be incorporated into clinical practice.

Materials and Methods: Cardiac function assessment has proven utility in the differential diagnosis of cardiomyopathies or prediction of perinatal mortality in congenital heart disease. In addition, some cardiac parameters with high sensitivity such as MPI or annular peak velocities have shown promising results in monitoring and predicting outcome in intrauterine growth restriction or congenital diaphragmatic hernia.

Conclusion: Cardiac function can be adequately evaluated in most fetuses when appropriate expertise, equipment and time are available. Fetal cardiac function assessment is a promising tool that may soon be incorporated into clinical practice to diagnose, monitor or predict outcome in some fetal conditions. Thus, more research is warranted to further define specific protocols for each fetal condition that may affect cardiac function.
\end{abstract}

Keywords: 4D STIC, echocardiography, fetal cardiac function, myocardial imaging, tissue Doppler.

\section{Importance of fetal cardiac function}

Recent advances in ultrasound have enabled the detection and characterisation of the physiology and pathophysiology of fetal circulation. Currently, the field of fetal cardiology is developing rapidly and fetal echocardiography is used not only for detection of structural anomalies ${ }^{1,2}$ but also to assess fetal cardiac function. ${ }^{3,4}$ Although fetuses rarely go into cardiac failure, the implementation of new cardiac technologies allows identifying subtle changes in cardiac function in subclinical phase. ${ }^{4,5}$ Heart failure is defined as the inability of the heart to supply sufficient blood flow to meet the body's needs ${ }^{6}$ and it is usually a late event that can be easily recognised by cardiomegaly, atrio-ventricular insufficiency and fetal hydrops. ${ }^{7}$ However, in the initial stages of an insult, the heart usually manages to adapt and there is a long subclinical period of cardiac dysfunction before end-stage heart failure. During this period of cardiac adaptation, changes in cardiac function, as well as in the heart's shape and size, can be determined. These changes are the heart's attempt to adapt to the insult, a process known as cardiac remodeling. ${ }^{8}$

Functional echocardiography has been demonstrated to help in the selection of high-risk populations and to be associated with outcome in several fetal conditions including intrauterine growth restriction, ${ }^{4,9}$ twin-to-twin transfusion syndrome, ${ }^{10,11}$ hydrops, $^{7}$ fetal arrhythmias, maternal diabetes ${ }^{12,13}$ and congenital diaphragmatic hernia. ${ }^{5,14}$ In addition, given the substantial evidence indicating the occurrence of programming of adult cardiovascular disease in fetal life, ${ }^{15-18}$ cardiac function assessment might help to predict perinatal and long-term cardiovascular outcomes. Despite fetal heart evaluation being challenging due to the small size of the heart, its high heart rate and restricted access to the fetus in the maternal abdomen, the evaluation of cardiac function is feasible in most fetuses by experienced healthcare professionals, whether they ultrasonographers, obstetricians, cardiologists, perinatologists or radiologists. Fetal echocardiography should be performed by specialists who are familiar with the prenatal diagnosis of fetal heart disease. ${ }^{1,2}$

The purpose of the following review is to describe the techniques currently available for performing a functional echocardiographic evaluation in the fetus, as well as some of the potential applications under research.

\section{Fetal cardiac physiology}

When performing fetal cardiac function assessment, it is important to keep in mind and understand the cardiac cycle in normal conditions. The primary function of the heart is to eject blood and provide adequate perfusion of organs. The main components initially involve the diastolic or ventricular filling stage with the movement of blood from the atria to the 


\section{longitudinal}
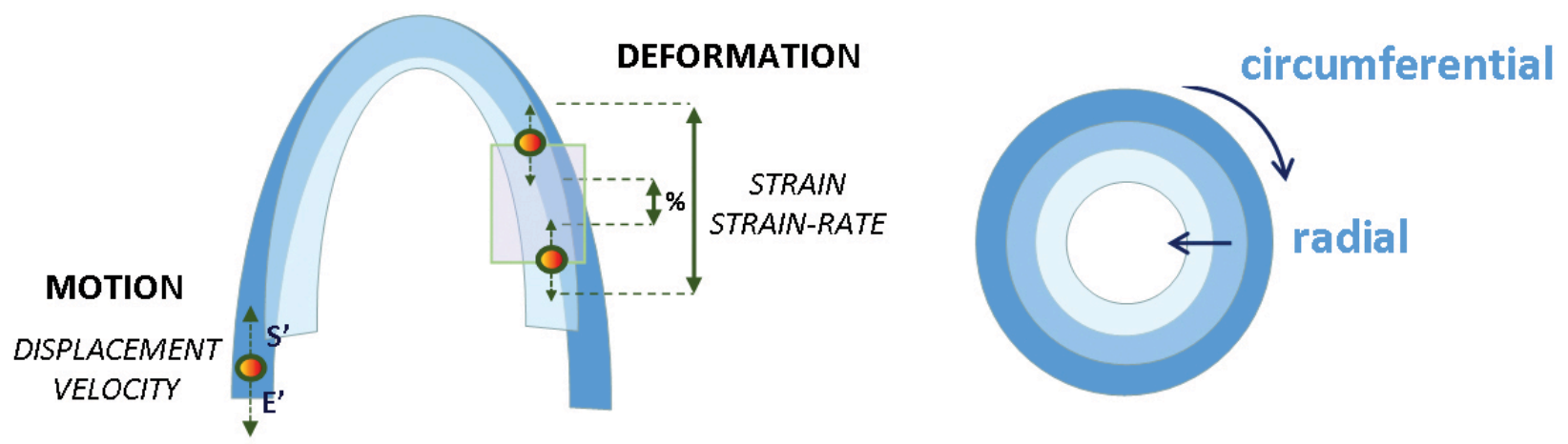

Figure 1: Graphic representation of the three-directional myocardial motility involving longitudinal, radial and circumferential contraction. The motion is shown as a single point motility determined by displacement and systolic ( $\mathrm{S}^{\prime}$ ) and early diastolic ( $\mathrm{E}^{\prime}$ ) annular peak velocities; and deformation by the change in length or thickness between two points represented as strain or strain rate.

ventricles; the systolic period involves blood movement from ventricles to the aorta and pulmonary arteries. The cardiac cycle can be further subdivided in five periods ${ }^{19}$ :

- Isovolumetric relaxation period: time period after the aortic/ pulmonary valve closure and opening of mitral/tricuspid valve. It is the first phase of diastole, while the myocardium starts relaxing, but the atrioventricular valves are still not open and therefore the blood is not entering into the ventricles yet.

- Early diastole: after the mitral/tricuspid valve opening, the blood from atria fills the ventricle in a passive manner due to pressure differences.

- Atrial contraction period (late diastole): the atria contracts and completes the filling of the ventricle.

- Isovolumetric contraction period: time period after the mitral/ tricuspid valves closure and before the opening of aortic/ pulmonary valves. It is the beginning of systole with the myocardium starting to contract but with the pressure inside the ventricle is not being high enough to open the aortic/ pulmonary valves.

- Ejection period: the ventricular pressure increases sufficiently to open aortic/pulmonary valves and blood is ejected from the ventricle.

Changes in cardiac function and shape are mainly determined by:

- Myocardial contractility defined as the ability of cardiac muscle to develop force for a given muscle length essentially during systole. ${ }^{20}$ Myocardial contractility can be evaluated by measuring motion or deformation with ultrasound. The distance covered by one point, determined by displacement and velocity, is defined as myocardial motion. The change in length or thickness of a segment (between two points) it is named deformation (Figure 1).

- Fiber orientation determined by the complex geometry of myocardial fibers involving longitudinal, radial and circumferential motion and deformation. ${ }^{21}$ (Figure 1).

- Volume and pressure loading conditions. Preload is the muscle length prior to contractility mainly determined by ventricular filling or blood volume in end-diastole. Afterload is the tension against which the ventricle must contract mainly determined by aorta/pulmonary artery pressure.
- Maturational changes that occur within the myocardium during gestation leading to changes in density, compaction of myofibrils, elasticity and myocardial contractility throughout pregnancy. ${ }^{22}$ Additionally, the fetal circulation is fed from the left and right ventricle in parallel, with three shunts - ductus venosus (DV), ductus arteriosus and foramen ovale - connecting right and left heart. The hemodynamic properties and functional ranges of these shunts are important determinants of the development of the fetal heart and circulation during the second and third trimesters.

\section{Technical considerations and limitations in the assessment of fetal cardiac function}

An optimal image of the heart is critical to adequately assess fetal cardiac structure and function. Ultrasound signal gain, image magnification, compound imaging, and harmonic imaging should be optimised. Sector, curvilinear, and endovaginal transducers can be used for this purpose. The transducer or scanner should be adjusted to operate at the highest clinically appropriate frequency, realizing that there is a trade-off between resolution and beam penetration. With modern equipment, fetal imaging studies performed from the anterior abdominal wall can usually use frequencies of $3.5 \mathrm{MHz}$ or higher, while scans performed from the vagina should be performed using frequencies of $5 \mathrm{MHz}$ or higher. ${ }^{1,2,23}$ Additionally, some specific techniques such as tissue Doppler usually require a $2-10 \mathrm{MHz}$ phased-array transducer.

Several intrinsic characteristics of the fetal heart and obstetric ultrasound equipments could affect the assessment of fetal cardiac function:

- Fetal echocardiography requires specific training and expertise to acquire images and interpreting results as well as adequate ultrasound equipment and cardiac software. ${ }^{1}$

- Maternal adiposity, oligoamnios or anterior placental location may interfere with the image quality.

- Intrinsic fetal particularities such as its position (fetal spine in anterior position), movements and size could hamper a complete evaluation. Longitudinal or radial motion requires an apical/basal or transverse view respectively.

- Smallness of the fetal heart reduces the accuracy of estimates of cardiac or vessel dimensions; in parameters that are estimates on the basis or formulas (e.g. cardiac output, ejection fraction) 


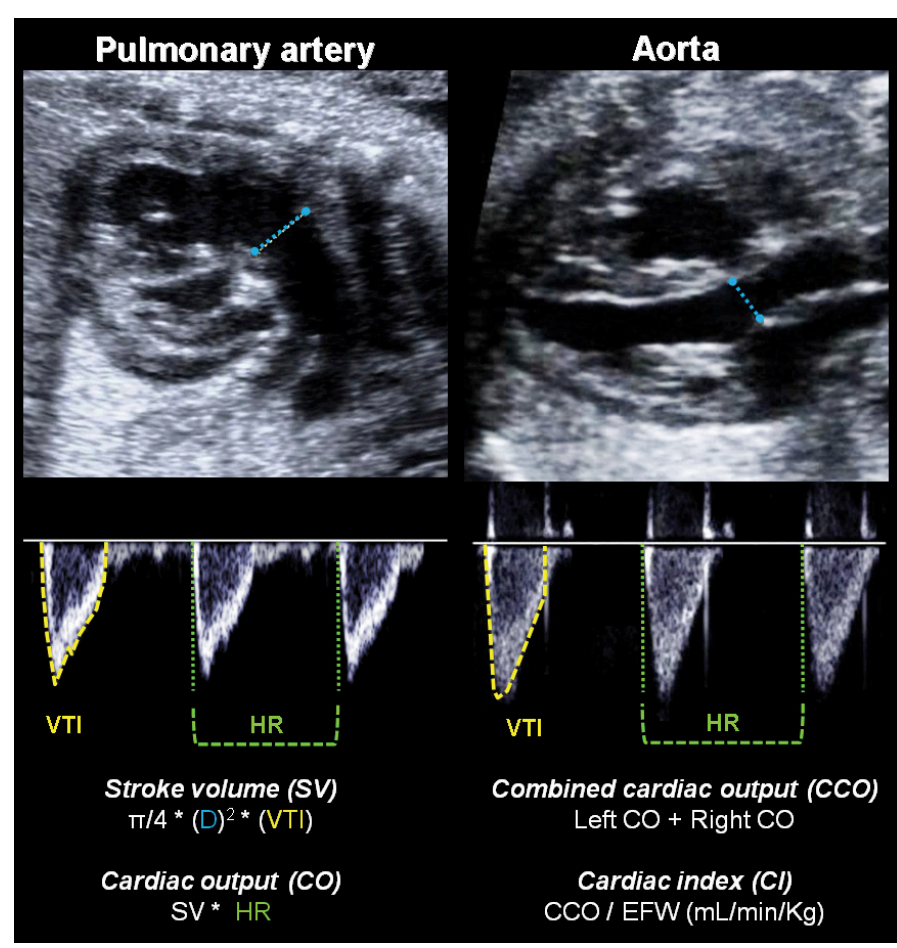

Figure 2: Illustration of the right and left ventricular outflow tracts for measuring stroke volume (SV) and cardiac output (CO). The valve diameter (D) is measured in a 2D image. Velocity time integral (VTI) of the blood flow and heart rate (HR) are evaluated in the spectral Doppler waveform. Combined cardiac output (CCO) is calculated by the sum of both $\mathrm{CO}$, and cardiac index $(\mathrm{Cl})$ represents the normalization by estimated fetal weight (EFW).

could show a wide variability as the error induced by one inaccurate dimension is multiplied in the final calculation. ${ }^{24}$

- Normality ranges throughout pregnancy are required to standardise and adjust measurements for fetal heart size. ${ }^{25}$

- The angle of acquisition is critical for these techniques, with most requiring an angle as close to $0^{\circ}$ as possible, while others such as cardiac dimensions or ejection fraction measurements are acquired perpendicular to the cardiac structure.

- A proper acquisition, processing and interpretation are required for offline analysis in techniques like $4 \mathrm{D}$ spatiotemporal image correlation (STIC), color tissue Doppler imaging (TDI) and 2D speckle tracking. ${ }^{26,27}$

- Most echocardiographic techniques are derived from parameters previously developed and validated in the adult heart. Electrocardiogram (ECG) being not possible to acquire in utero limit post-processing of offline techniques (dummy ECG will be required). Changes in fetal cardiomyocyte maturation and the fetal circulation pattern differ from that in the adult, and these patterns may also change during pregnancy.

- Some techniques used in fetal functional echocardiography have not been validated yet, limiting their interpretation.

\section{Techniques for the assessment of fetal cardiac function}

A broad range of ultrasound techniques have been applied for evaluation of fetal cardiac function including the fundamental image (2D image), M-mode, conventional and tissue Doppler, $2 \mathrm{D}$ speckle tracking and $4 \mathrm{D}-\mathrm{STIC}{ }^{26,27}$ A description of the most common techniques used in the fetus follows.

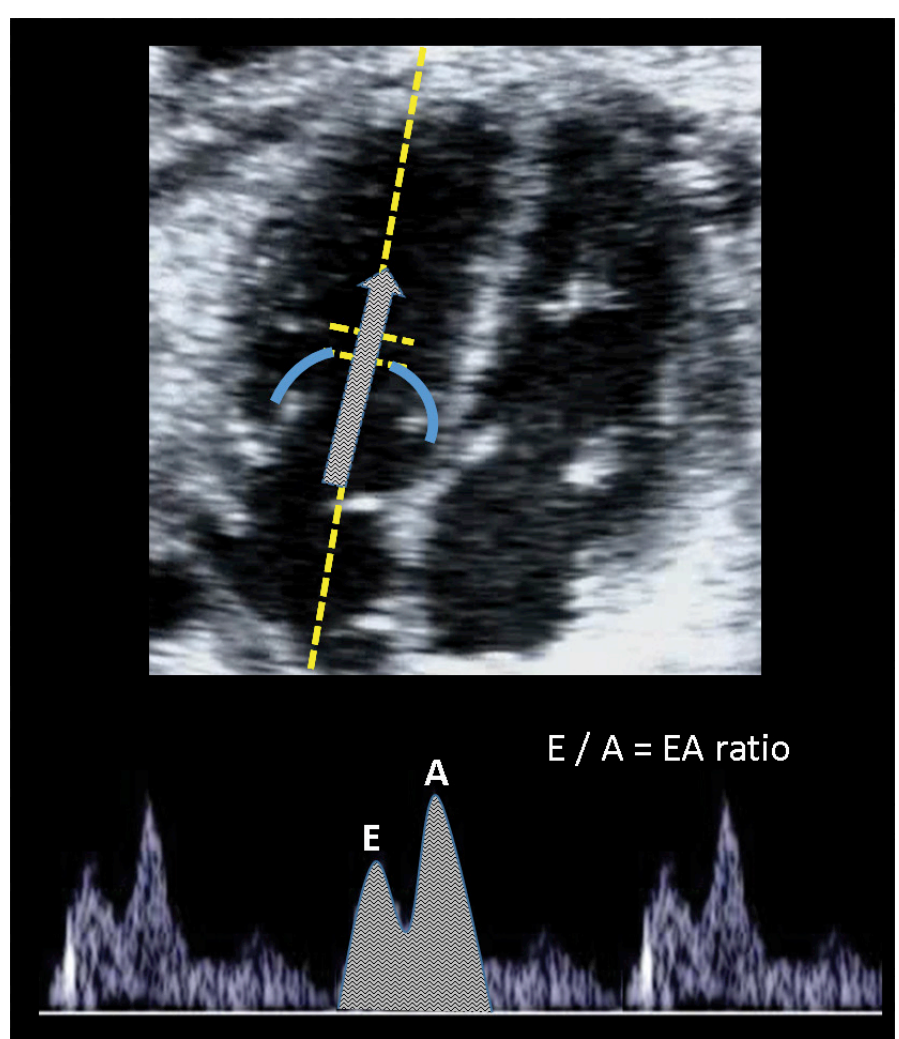

Figure 3: Image of the E/A ratio evaluation. The sample gate is placed just below the atrio-ventricular valves in a four-chamber view in order to display biphasic inflow (including the $E$ (early diastole) and $A$ (atrial contraction).

\section{D-imaging}

A comprehensive evaluation of the fetal heart anatomy and structure using 2D-imaging is crucial to confirm the structural integrity and also look for signs of remodeling. Cardiac remodeling is defined as changes in size, shape, structure and physiology of the heart after an injury. So, for example, in the case of recipients of twin-to-twin transfusion syndrome there is pressure and volume overload which conditionates cardiomegaly and myocardial hypertrophy that can be evaluated and measured just by using $2 \mathrm{D}$ images. Images in $2 \mathrm{D}$ are also used to perform measurements of most cardiac dimensions such as atrial size, valve diameters or ventricular dimensions.

\section{Conventional Doppler}

Conventional Doppler can be applied to obtain information about the blood flow through the heart. It allows measuring ventricular blood outflow (systole) and inflow (diastole) as well as calculation of time periods.

Systolic function can be evaluated by measuring the outflow from the ventricles (Figure 2). Diameters of the aortic and pulmonary valves are obtained in frozen real-time images during early to mid-systole by the leading-edge-to-edge method. Velocity-time integrals are calculated by manual trace of the spectral Doppler area and can be multiplied by the area of the outflow wave to calculate the stroke volume which represents the amount of blood ejected per heartbeat. ${ }^{19}$ Combining this information with the fetal heart rate allows estimating the left and right cardiac output. In the fetus, the sumatory of both is 


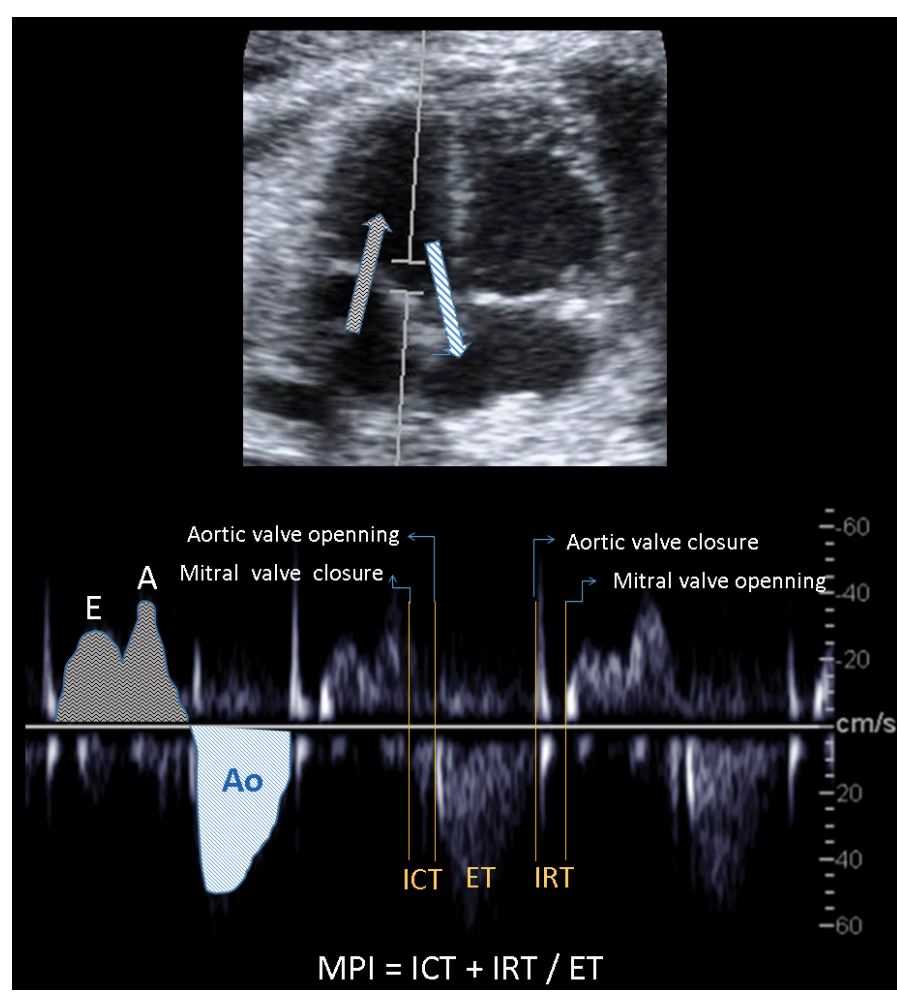

Figure 4: Illustration of myocardial performance index (MPI) assessment by spectral Doppler. Placing the Doppler sample volume in a four-chamber view on the medial wall of the ascending aorta, the mitral biphasic inflow (grey arrow, early $(\mathrm{E})$ and atrial $(\mathrm{A})$ waveforms) and the aortic outflow (blue arrow and waveform $\left(A_{0}\right)$ ) are displayed in the same spectral image. The MPI is calculated by measuring time intervals including: isovolumic contraction time (ICT) from the closure of the mitral valve to the opening of the aortic valve; ejection time (ET) from the opening to closure of the aorta; and isovolumic relxaxation time (IRT) from the closure of the aortic valve to the opening of the mitral valve.

named the combined cardiac output, which should normally be expressed as the cardiac index (cardiac output divided by estimated fetal weight). ${ }^{24}$

The E/A ratio reflect the ventricular inflow pattern (Figure 3 ) and it is used to evaluate diastolic function or ventricular relaxation. It is evaluated by the spectral Doppler sample volume below the atrioventricular (AV) valves, where a biphasic wave is displayed in the normal fetus. The first wave component, the $\mathrm{E}$ wave (early or passive diastole) represents myocardial relaxation and negative pressure of the ventricle. The A wave (active, atrial or late diastole) represents the atrial contraction during ventricular filling. The ratio is obtained by the division of the peak velocities of the E over the A waveform and usually the value is $<1 .^{28} \mathrm{E} / \mathrm{A}$ velocities increase as pregnancy progresses ${ }^{29}$ with higher velocities in the tricuspid valve as compared to the mitral. ${ }^{30,31}$

The myocardial performance index (MPI or Tei Index) is considered a marker of global cardiac function and provides information on the different time periods during both systolic and diastolic phases explained above. These time periods are the isovolumetric contraction time (ICT), the ejection time (ET) and isovolumetric relaxation time (IRT) (Figure 4). The MPI is usually measured in a cross section of the fetal thorax at the 4-chamber view, placing the Doppler sample volume on the medial wall of the ascending aorta including the aortic and

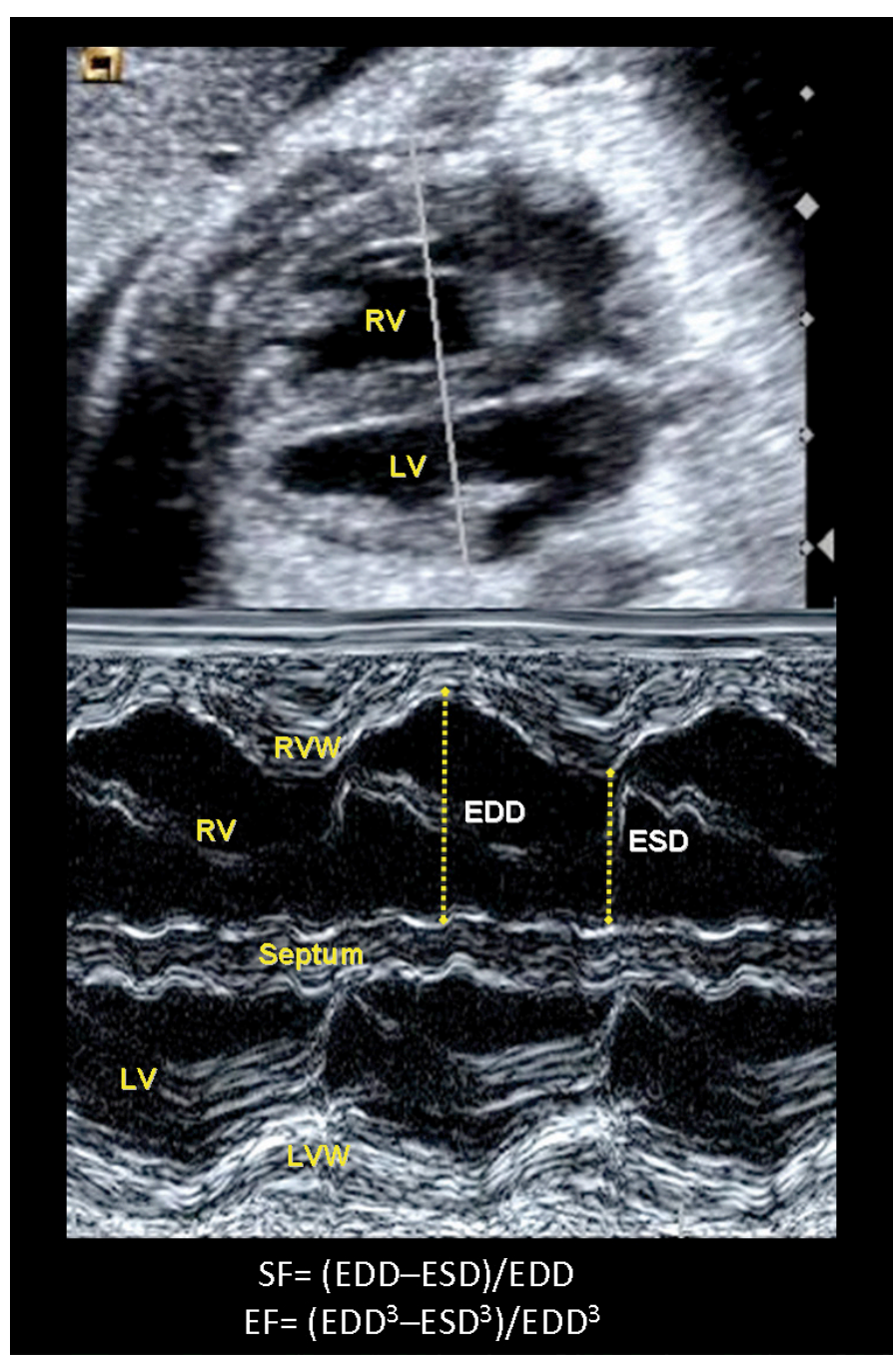

Figure 5: Illustration of a transverse four-chamber view in order to measure shortening (SF) and ejection fractions (EF) of the right (RV) and left ventricles (LV) by M-mode. The arrows between the septal and right free walls show the measurement of end-diastolic (EDD) and end systolic (ESD) diameters required for the SF and EF calculation. RVW= right ventricle wall; LVW= left ventricle wall.

mitral valve. The valvular clicks in the Doppler wave can be used as landmarks to calculate the three time periods. ${ }^{32}$ ICT is measured from the closure of the mitral valve to the opening of the aortic valve, ET from the opening to closure of the aorta and IRT from the closure of the aortic valve to the opening of the mitral valve. MPI is calculated as (ICT + IRT) / ET. Normal values are increasing throughout gestation. ${ }^{32-35}$

\section{M-mode}

M-mode can be applied for the evaluation of longitudinal and radial motion of cardiac structures including valve annuli or myocardial walls. It can also be used to evaluate cardiac dimensions such as wall thickness or chamber size.

By assessing the short-axis motion of the heart (radial function), shortening and ejection fractions can be calculated (Figure 5). The beam is positioned along the short axis of the heart in the four-chamber view (transverse view), perpendicular to the interventricular septum. ${ }^{36}$ Using this view, M-mode can be applied to obtain measurements for the end-systolic (ESD) 


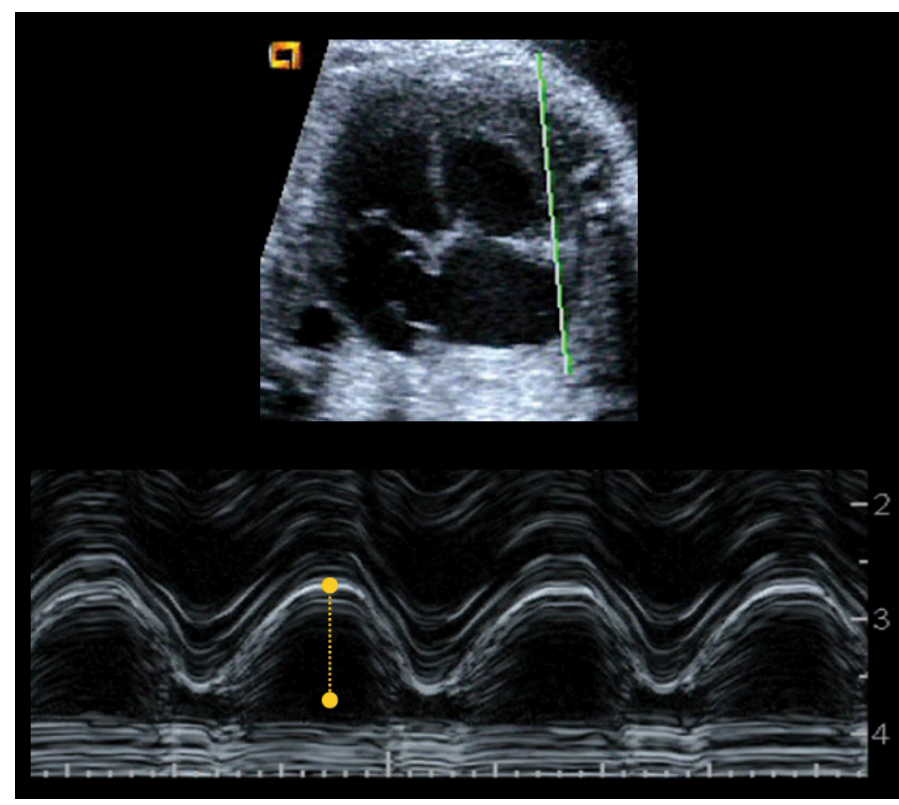

Figure 6: Illustration of the tricuspid annular plane systolic excursion (TAPSE) measurement by applying M-mode at the tricuspid valve annulus in an apical four-chamber view.

and end-diastolic diameters (EDD) used to calculate shortening fraction and ejection fraction by applying Teicholz's formula. ${ }^{37,38}$ Shortening fraction is defined as the percentage the ventricular diameters shorten during contraction and it is calculated as: (EDD-ESD)/EDD. ${ }^{39}$ Ejection fraction is defined as the percentage of blood ejected in each heart cycle. In order to calculate it the diameters are elevated to a cube to estimate ventricular volumes as: $\left(\mathrm{EDD}^{3}-\mathrm{ESD}^{3}\right) / \mathrm{EDD}^{3}$. Normal values in the fetus have been published. ${ }^{36}$ Shortening and ejection fractions usually altered only in late stages of deterioration, as it mainly reflects radial function. ${ }^{37}$

M-mode can be also applied in an apical or basal 4-chamber view in order to evaluate the long-axis motion of the heart. This approach is most suited to right ventricle examination owing to the longitudinal nature for right ventricle muscle fibers as opposed to the mainly circumferential orientation of the left ventricle muscle fibers. An apical or basal four-chamber view is used to measure the maximum excursion of the area of junction between the tricuspid annulus and the right ventricle free wall from end-diastole to end-systole (referred as tricuspid annular plane systolic excursion, TAPSE) (Figure 6). It can also be applied to mitral annulus (MAPSE).$^{40}$ Normal ranges for fetus are published ${ }^{41}$ and recently described as a very sensitive parameter for assessing cardiac dysfunction in several fetal conditions such as fetal growth restriction. ${ }^{42}$

\section{TDI}

TDI is a reproducible echocardiographic technique that uses frequency shifts in ultrasound waves to calculate myocardial velocity, which is characterise $\mathrm{d}$ by a lower velocity and higher amplitude. TDI can be applied online (spectral TDI) to evaluate annular velocities or offline (color-coded TDI) to assess myocardial motion and deformation.

Spectral TDI can be applied in an apical o basal 4-chamber view to assess longitudinal motion (Figure 7). The sample volume $(2-4 \mathrm{~mm})$ is usually applied in the basal part of the ventricle or

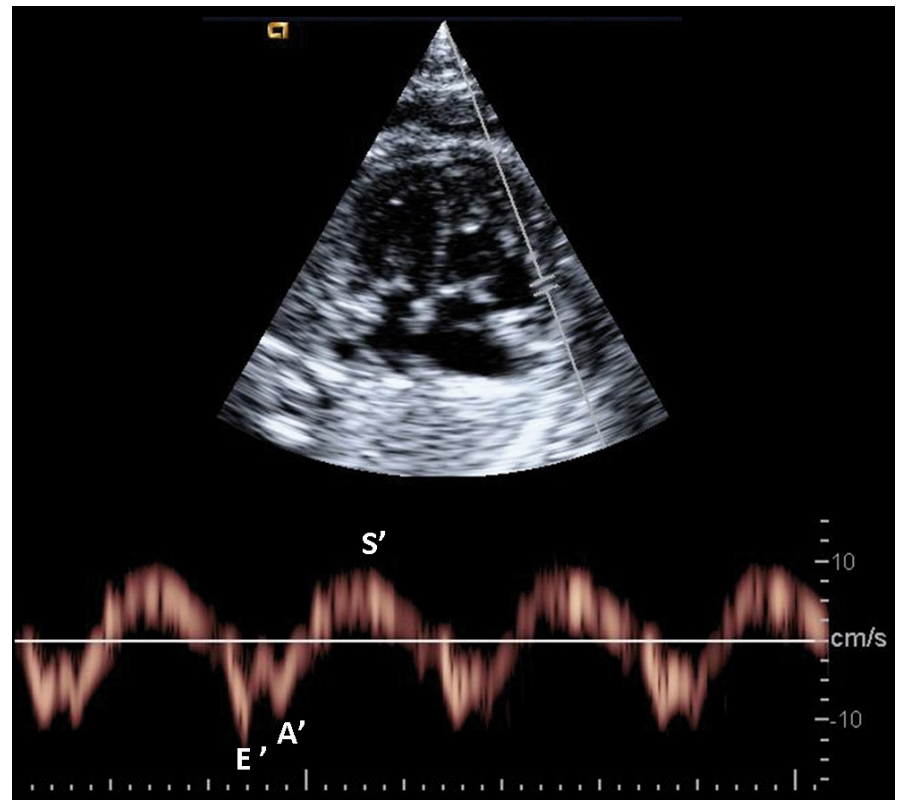

Figure 7: Example of early $\left(E^{\prime}\right)$ and late $\left(A^{\prime}\right)$ diastolic and systolic $\left(S^{\prime}\right)$ peak annular velocities obtained by spectral tissue Doppler at the right annulus.

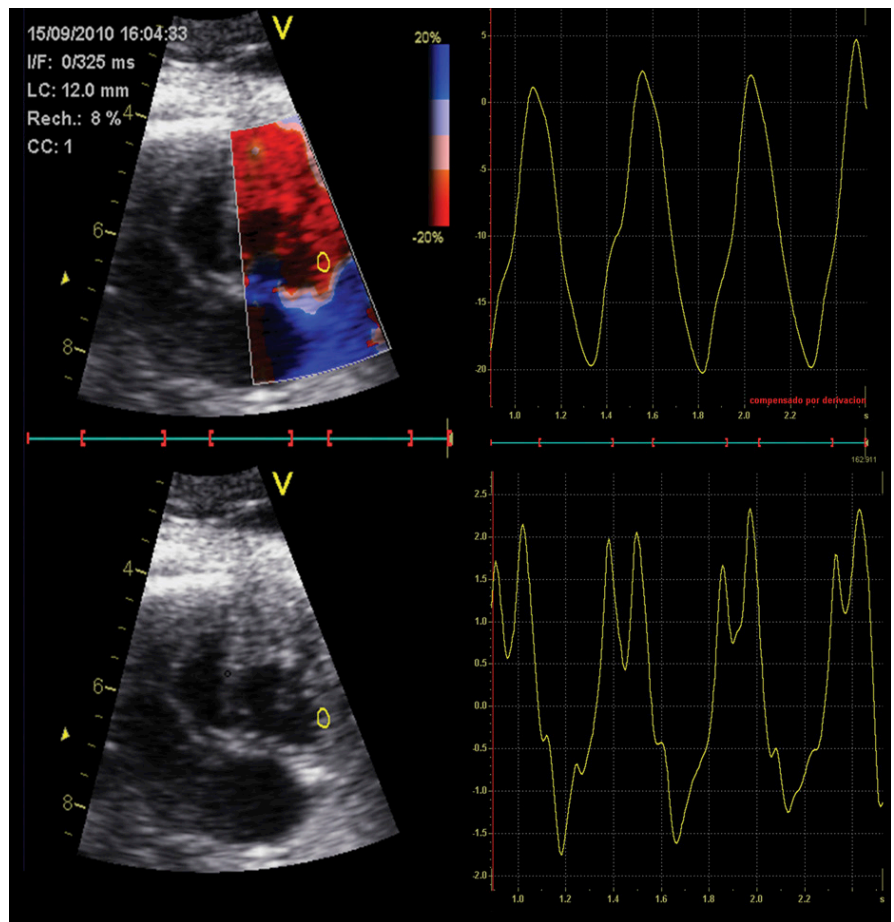

Figure 8: Offline analysis of strain (above) and strain rate (below) waveforms at the right basal free wall using color tissue Doppler.

annulus with an insonation angle $<30^{\circ}$. The velocity of annular movement toward the Doppler cursor is displayed as a waveform moving towards the atria. ${ }^{43}$ The peak annular velocities obtained are E' or Ea (early diastolic annular peak velocity), A' or Aa (latediastolic annular peak velocity) and $S^{\prime}$ or Sa (systolic annular peak velocity) during the ventricular systole. Spectral TDI also allows the calculation of time periods in order to measure the myocardial performance index (MPI') as: (ICT' + IRT')/ET'44. TDI has been shown to be feasible in fetuses ${ }^{45}$ and normal ranges have been published for both velocities and MPI'25. Peak annular 


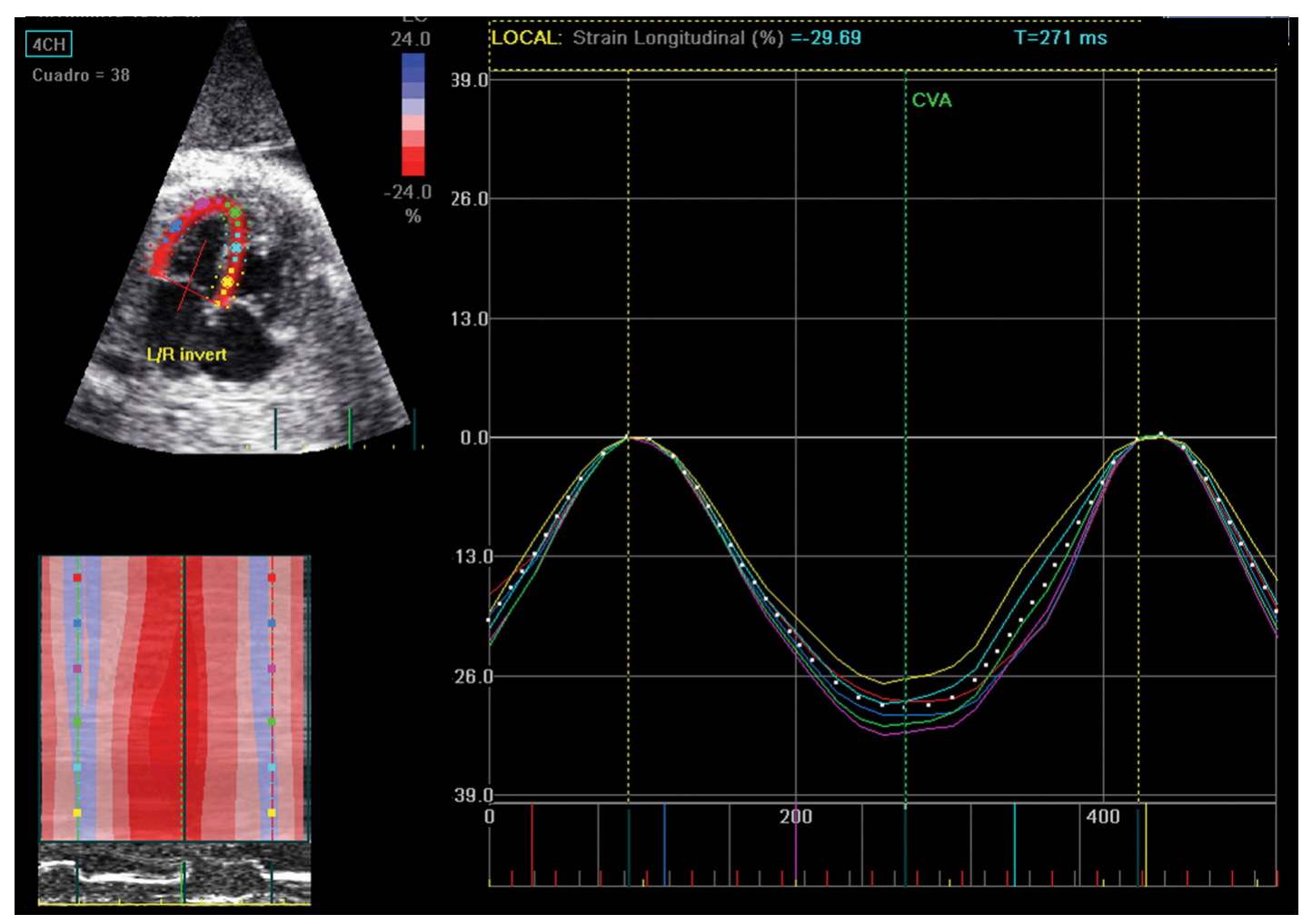

Figure 9: Offline analysis of regional and global strain waveforms of the left ventricle wall using $2 \mathrm{D}$ speckle tracking. velocities have been demonstrated to early and sensitive markers of cardiac dysfunction in several fetal conditions. ${ }^{46,47}$

Color TDI clips require a complex offline analysis in order to evaluate myocardial deformation (Figure 8). An optimal clip acquisition is critical for obtaining reliable measurements. A cardiac cine loop of an apical or basal 4-chamber view (at least 5 seconds of non-compressed data) should be acquired during fetal quiescence. It is critical to acquire images at high frame rate ( $>$ 200 frames per second) that could be achieved with a narrow 2D scan area and TDI color box. Strain represents the lengthening change of a myocardial segment from its original length, while strain rate consists of the change of strain during time. Negative strain values described shortening (usually in systole), and positive ones describe lengthening (usually in diastole) relative to the instantaneous dimension. A color-coded representation of myocardial velocities, strain and strain-rate can be displayed offline through three to five cardiac cycles. It has been recently reported an acceptable feasibility and reproducibility for the fetal heart by using a dummy ECG by manually indicating the onset of each cardiac cycle in the $2 \mathrm{D}$ clip based on valve motion. ${ }^{48}$ These parameters are also early markers of global and regional cardiac function by providing information on myocardial deformation and interaction with neighboring segments. ${ }^{47}$

\section{D speckle tracking techniques}

Recent reports have described the use of non-Doppler technology for assessing fetal cardiac function. 2D speckle tracking techniques allow myocardial motion and deformation to be quantified by using frame-by-frame tracking of bright myocardial areas (speckles). ${ }^{49}$ This angle-independent method requires post-processing and off-line analysis of $2 \mathrm{D}$ images and allows myocardial strain and strain rate to be measured (Figure 9). Most speckle tracking techniques are semi-automated, but the user is responsible for drawing the tracking line defining the endocardial border of the ventricle from the 4-chamber view, placing it along the endocardial border, apex and atrioventricular valve annulus in a single frame of a stored heart cycle. The software automatically tracks the border between the cavity and the endocardium in subsequent frames; due to the absence of an ECG, the cardiac cycle is determined from mechanical movements: the mitral valve movement to detect systole and diastole. ${ }^{50}$ Depending on sonographic view selected, the software divides the tracking line into corresponding myocardial segments providing either global (average values for the whole tracking line) or segmental strain. This technique has been also recently validated for the fetal heart of applied with strict methodological criteria including a high quality/high-frame rate $2 \mathrm{D}$ clip acquisition and the use of dummy ECG. ${ }^{48}$

\section{D-STIC}

4D-STIC is based on a sweep (volume data set) of the fetal heart containing a complete reconstructed cardiac cycle allowing a 3D reconstruction of the fetal heart over time. ${ }^{51}$

$4 \mathrm{D}-$ STIC has been proposed for measuring ventricular volumes allowing a potentially more accurate estimation of cardiac output and ejection fraction. ${ }^{37,52,53}$ By scrolling through a saved STIC volume, the operator can obtain the 4-chamber view at end-diastole and end-systole and then perform a manual or semi-automated volume measurement of the cardiac ventricles. Virtual organ computerised analysis (Figure 10) or inversion mode can be applied to obtain the desired volumes. The goal of obtaining ventricular volumes is to extrapolate cardiac functional measurements like stroke volume, ejection fraction and cardiac output. ${ }^{54}$ The various combinations of modalities and their subcommands have been compared for feasibility and reproducibility. ${ }^{55} 4 \mathrm{D}$-STIC is a promising technique that 


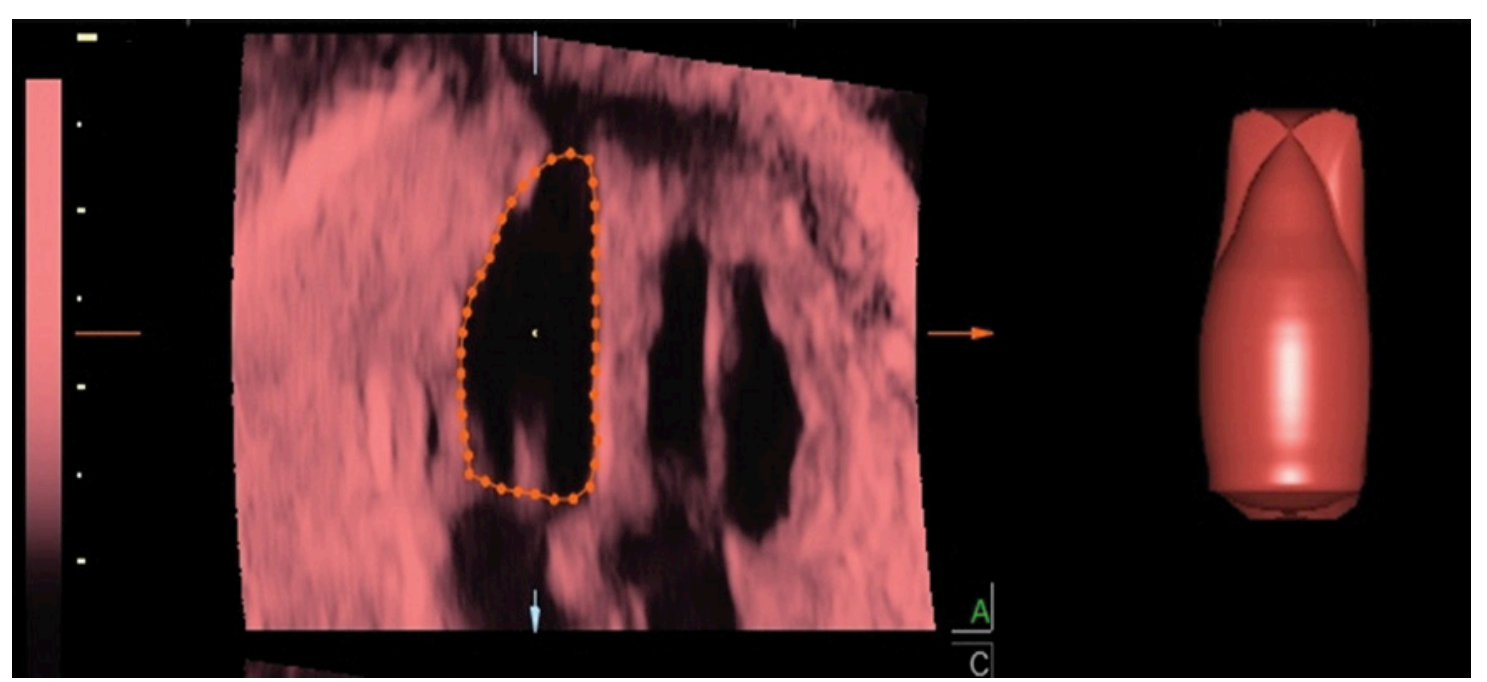

chronic adaptation

\section{subclinical cardiac dysfunction}

\section{longitudinal}

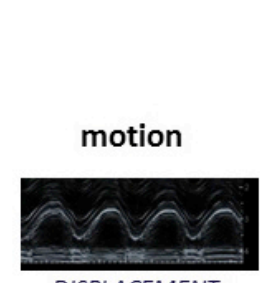

DISPLACEMENT

VELOCITY

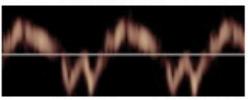

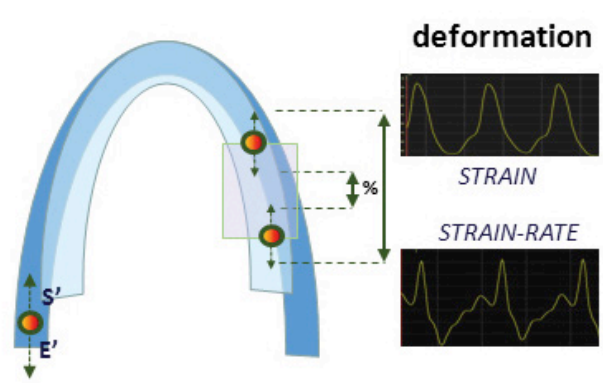
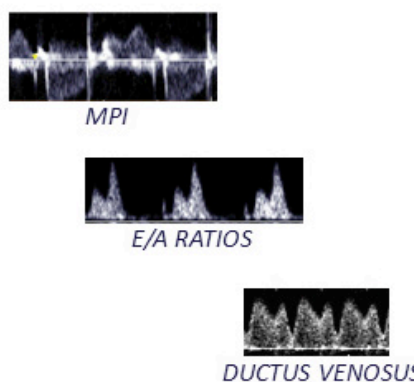

diastolic
DUCTUS VENOSUS

systolic
Figure 10: Postprocessing analysis of the left ventricular volume through virtual organ computerized analysis using 4D-spatio temporal correlation.

Figure 11: Graphic representation of cardiac function parameters at the different stages of the fetal adaptation to disease, illustrating longitudinal and diastolic function becoming abnormal at earlier stages than radial and systolic. Ductus venosus, E/A ratios, myocardial performance index (MPI) and cardiac output are measured by conventional Doppler; ejection fraction and longitudinal displacement by M-mode; systolic ( $\mathrm{S}^{\prime}$ ) and early diastolic ( $\left.E^{\prime}\right)$ annular peak velocities by spectral tissue Doppler; and strain and strain-rate by offline tissue Doppler or 2D speckle tracking. requires further studies to improve its applicability in fetal cardiac function assessment. ${ }^{37}$

It can also be used to offline apply M-mode and measure long-axis motion (TAPSE and MAPSE). M-mode STIC allows adjustment of the wave pattern through the modification of image orientation in order to optimise the image by rotating the volume to obtain the desired plane.

\section{Clinical and research applications of fetal cardiac function assessment}

The most suitable parameters for assessing fetal cardiac function will be mainly determined by the cause of the cardiac dysfunction. Abnormal values of ejection fraction or cardiac output are usually found in late stages of deterioration, and therefore more sensitive parameters have been proposed for earlier diagnosis and monitoring of fetal cardiac dysfunction (Figure 11). Parameters reflecting longitudinal function (such as annular displacement or velocities) are typically affected in the very early stages as compared to radial function (such as ejection fraction). Similarly, diastolic parameters (such as DV or IRT) are the first to be altered, reflecting impaired relaxation and compliance due to a stiffer or less effective heart. Fetal cardiac function assessment has been proposed to help in the diagnosis, monitoring or prognosis in several fetal conditions including primary cardiac disease or extracardiac anomalies:

- Primary cardiac disease: Various types of congenital heart disease show specific differences in volume and/or pressure load, as well as distinct degrees of cardiac remodeling and 
adaptation. Fetal cardiac function has been proposed as selection criteria to predict progression to hypoplasticleftheart syndrome and eligibility for prenatal cardiac intervention in critical aortic stenosis. ${ }^{44}$ Functional echocardiography can be also useful for the better understanding and prognosis of other congenital heart diseases or primary cardiomyopathies.

- Cardiac adaptation to extracardiac anomalies: The heart is a central organ in fetal adaptive mechanisms to disease, and cardiac dysfunction (due to heart compression, volume/ pressure overload or direct insult to the myocardium) is recognised as being among the central pathophysiologic features of intrauterine growth restriction, ${ }^{25,50}$ maternal diabetes, ${ }^{45,56}$ twin-to-twin transfusion syndrome, ${ }^{10}$ congenital diaphragmatic hernia $(\mathrm{CDH})^{5,14}$ and other fetal anomalies such as congenital cystic adenomatoid malformation, sacrococcygeal teratoma or arteriovenous malformations, Dows' syndrome, absence of the DV, lower urinary tract obstruction with massive bladder distension, fetal anemia and preterm rupture of membranes, particularly in cases with intra-amniotic infection. ${ }^{45,46}$

\section{Conclusions}

Fetal heart evaluation is feasible and reproducible, although challenging due to the smallness of the heart, the high heart rate and limited access to the fetus. Fetal cardiac function may be affected by several of cardiac and extracardiac anomalies. ${ }^{3-5,7}$ Although research efforts have recently recognised the potential value of monitoring cardiac function for clinical management, most parameters are still in the research phase. The exception is $\mathrm{DV}$, which is already being used in clinical practice for staging twin-to-twin transfusion syndrome $e^{57}$ or monitoring earlyonset intrauterine growth restriction. ${ }^{9,58}$ Another example is the integration of some cardiac function parameters (mitral inflow and myocardial contractility) in the inclusion criteria for fetal valvuloplasty in critical aortic stenosis. ${ }^{57,59}$ Additionally, some cardiac parameters have already shown a strong correlation with outcome and may soon be incorporated into clinical practice. For example, cardiac function assessment has proven utility in the differential diagnosis of cardiomyopathies ${ }^{60}$ or prediction of perinatal mortality in congenital heart disease. ${ }^{61}$ Similarly, first-trimester DV and tricuspid regurgitation assessment has an additional predictive value for Down syndrome and congenital heart disease. ${ }^{62}$ In addition, some cardiac parameters with high sensitivity such as MPI or annular peak velocities have shown promising results in monitoring and predicting outcome in intrauterine growth restriction ${ }^{9,58}$ or congenital diaphragmatic hernia. ${ }^{5}$ Other promising parameters that have recently been proposed remain to be validated in fetal heart assessment. Conversely, cardiac function assessment does not have demonstrated utility in the staging or prognosis of twin-to-twin transfusion syndrome. ${ }^{11}$

Complete assessment and integration of the distinct components of systolic and diastolic function are crucial, as no single simple test can fully evaluate fetal cardiac function. Several groups have suggested scoring systems, ${ }^{3,7}$ which combine methods of assessment to provide a more global overview of cardiac function and which could be used as a prognostic marker or to define heart failure. Although scoring may be superior to single measurements in defining compromised cardio-circulatory function, there are still many limitations to the incorporation of the proposed scores in clinical practice. Scores need to be validated not only to predict perinatal mortality but also to predict morbidity and long-term outcome. Many definitions of abnormal cardiac function need to be standardised. Cardiac function can be affected by various fetal abnormalities through volume or pressure overload, hypoxia, hyperglycemia, heart compression, direct myocardial damage, etc. Although disease-specific cardiovascular profile scores would most probably improve the predictive value for adverse outcome, constructing such a profile would be highly complex. Finally, the already proposed scores include traditional cardiac parameters, while new parameters such us MPI, ${ }^{63}$ annular peak velocities ${ }^{46}$ or ventricular strain ${ }^{50}$ have been demonstrated to be more sensitive in certain specific conditions. Therefore, studies to further evaluate the potential utility of recently proposed techniques and to define the best combination of parameters are warranted. New cardiovascular parameters should be incorporated with caution, taking into account all the potential limitations of fetal echocardiography.

Despite these limitations, cardiac function can be adequately evaluated in most fetuses when appropriate expertise, equipment and time are available. Fetal cardiac function assessment is a promising tool that may soon be incorporated into clinical practice to diagnose, monitor or predict outcome in some fetal conditions. Thus, more research is warranted to further define specific protocols for each fetal condition that may affect cardiac function.

\section{Funding sources}

This review was supported by grants from Instituto de Salud Carlos III and Ministerio de Economia y Competitividad (ref. PI11/00051, PI11/01709, PI12/00801 and SAF2012-37196) cofinanciado por el Fondo Europeo de Desarrollo Regional de la Unión Europea "Una manera de hacer Europa", the Fundación Mutua Madrileña, Obra Social "la Caixa”, and Cerebra Foundation for the Brain Injured Child (Carmarthen, Wales, UK).

\section{References}

1 Carvalho JS, Chaoui R, Copel JA, DeVore GR, Hecher K, Lee W, et al. ISUOG practice guidelines (updated): sonographic screening examination of the fetal heart. Ultrasound Obstet Gynecol 2013; (41): 348-59.

2 Lee W, Allan L, Carvalho JS, Chaoui R, Copel J, Devore G, et al. ISUOG consensus statement: what constitutes a fetal echocardiogram? Ultrasound Obstet Gynecol 2008; 32 (2): 239-42.

3 Rychik J, Tian Z, Bebbington M, Xu F, McCann M, Mann S, et al. The twin-twin transfusion syndrome: spectrum of cardiovascular abnormality and development of a cardiovascular score to assess severity of disease. Am J Obstet Gynecol 2007; 197 (4): 392. e1-8.

4. Crispi F, Hernandez-Andrade E, Pelsers MM, Plasencia W, BenavidesSerralde JA, Eixarch E, et al. Cardiac dysfunction and cell damage across clinical stages of severity in growth-restricted fetuses. Am J Obstet Gynecol 2008; 199 (3): 254. e1-8.

5 Van Mieghem T, Gucciardo L, Done E, Van Schoubroeck D, Graatsma EM, Visser GH, et al. Left ventricular cardiac function in fetuses with congenital diaphragmatic hernia and the effect of fetal endoscopic tracheal occlusion. Ultrasound Obstet Gynecol 2009; 34 (4): 424-29.

6 Jessup MA, Casey DE, Feldman AM, Francis GS, Ganiats TG, 
Konstam MA, et al. 2009 focused update: ACCF/AHA Guidelines for the Diagnosis and Management of Heart Failure in Adults: a report of the American College of Cardiology Foundation/ American Heart Association Task Force on Practice Guidelines: developed in collaboration with the International Society for Heart and Lung Transplantation. Circulation 2009; 14 (119): 1977-2016.

7 Huhta JC. Guidelines for the evaluation of heart failure in the fetus with or without hydrops. Pediatr Cardiol 2004; 25 (3): 274-86.

8 Opie LH, Commerford PJ, Gersh BJ. MA. P. Controversies in ventricular remodelling. Lancet 2006; 367: 356-67.

9 Figueras F, Puerto B, Martinez JM, Cararach V, Vanrell JA. Cardiac function monitoring of fetuses with growth restriction. Eur J Obstet Gynecol Reprod Biol 2003; 110 (2): 159-63.

10 Karatza AA, Wolfenden JL, Taylor MJ, Wee L, Fisk NM, Gardiner HM. Influence of twin-twin transfusion syndrome on fetal cardiovascular structure and function: prospective case-control study of 136 monochorionic twin pregnancies. Heart 2002; 88 (3): 271-77.

11. Stirnemann JJ, Mougeot M, Proulx F, Nasr B, Essaoui M, Fouron JC, et al. Profiling fetal cardiac function in twin-twin transfusion syndrome. Ultrasound Obstet Gynecol; 35 (1): 19-27.

12 Gardiner HM, Pasquini L, Wolfenden J, Kulinskaya E, Li WMH. Increased periconceptional maternal glycated haemoglobin in diabetic mothers reduces fetal long axis cardiac function. Heart 2006; 92: 1125-30.

13 Turan S, Turan OM, Miller J, Harman C, Reece EA, et al. Decreased fetal cardiac performance in the first trimester correlates with hyperglycemia in pre-gestational maternal diabetes. Ultrasound Obstet Gynecol 2011; 38: 325-31.

14. Stressig R, Fimmers R, Eising K, Gembruch U, Kohl T. Preferential streaming of the ductus venosus and inferior caval vein towards the right heart is associated with left heart underdevelopment in human fetuses with left-sided diaphragmatic hernia. Heart; 96 (19): 1564-8.

15 Barker DJ, Osmond C, Golding J, Kuh D, Wadsworth ME. Growth in utero, blood pressure in childhood and adult life, and mortality from cardiovascular disease. BMJ 1989; 298 (6673): 564-67.

16 Hennington BS, Alexander BT. Linking IUGR and Blood Pressure: Insight into the Human Origins of Cardiovascular Disease. Circulation 2013; Epub ahead of print.

17 Bjarnegard N, Morsing E, Cinthio M, Lanne T, Brodszki J. Cardiovascular function in adulthood following intrauterine growth restriction with abnormal fetal blood flow. Ultrasound Obstet Gynecol 2013; 41 (2): 177-84.

18 Brodszki J, Lanne T, Marsal K, Ley D. Impaired vascular growth in late adolescence after intrauterine growth restriction. Circulation 2005; 111 (20): 2623-28.

19 Guyton AC. HJ. Textboook of Medical Phisiology. 12 ed. Philadelphia: Elsevier Saunders 2011.

20 Bijnens B, Cikes M, Butakoff C, Sitges M, Crispi F. Myocardial motion and deformation: What does it tell us and how does it relate to function? Fetal Diagn Ther 2012; 32 (1-2): 5-16.

21 Anderson RH, Smerup M, Sanchez-Quintana D, Loukas M, Lunkenheimer PP. The three-dimensional arrangement of the myocytes in the ventricular walls. Clin Anat 2009; 22 (1): 64-76.

22 Tobita K, Garrison JB, Liu LJ, Tinney JP, Keller BB. Threedimensional myofiber architecture of the embryonic left ventricle during normal development and altered mechanical loads. Anat Rec A Discov Mol Cell Evol Biol 2005; 283 (1): 193-201.

23 AIUM practice guideline for the performance of fetal echocardiography. J Ultrasound Med 2011; 30 (1): 127-36.

24 Hernandez-Andrade E, Benavides-Serralde JA, Cruz-Martinez R, Welsh A, Mancilla-Ramirez J. Evaluation of conventional Doppler fetal cardiac function parameters: E/A ratios, outflow tracts, and myocardial performance index. Fetal Diagn Ther; 32 (1-2): 22-29.

25 Comas M, Crispi F, Gomez O, Puerto B, Figueras F, Gratacos E. Gestational age and estimated fetal weight-adjusted reference ranges for myocardial tissue Doppler indices at 24-41 weeks' gestation. Ultrasound Obstet Gynecol 2011; 37 (1): 57-64.

26 Comas M, Crispi F. Assessment of fetal cardiac function using tissue Doppler techniques. Fetal Diagn Ther 2012; 32 (1-2): 30-38.

27 Germanakis I, Gardiner H. Assessment of fetal myocardial deformation using speckle tracking techniques. Fetal Diagn Ther 2012; 32 (1-2): 39-46.

28 Carceller-Blanchard AM, Fouron JC. Determinants of the Doppler flow velocity profile through the mitral valve of the human fetus. $\mathrm{Br}$ Heart J 1993; 70 (5): 457-60.

29 Tulzer G, Khowsathit P, Gudmundsson S, Wood DC, Tian ZY, Schmitt $\mathrm{K}$, et al. Diastolic function of the fetal heart during second and third trimester: a prospective longitudinal Dopplerechocardiographic study. Eur J Pediatr 1994; 153 (3): 151-54.

30 van der Mooren K, Barendregt LG, Wladimiroff JW. Fetal atrioventricular and outflow tract flow velocity waveforms during normal second half of pregnancy. Am J Obstet Gynecol 1991; 165 (3): 668-74.

31 Rizzo G, Pietropolli A, Capponi A, Cacciatore C, Bufalino L, Arduini $\mathrm{D}$, et al. Echocardiographic studies of the fetal heart. $J$ Perinat Med 1994; 22 (Suppl 1): 46-50.

32 Hernandez-Andrade E, Figueroa-Diesel H, Kottman C, Illanes S, Arraztoa J, Acosta-Rojas R, et al. Gestational-age-adjusted reference values for the modified myocardial performance index for evaluation of fetal left cardiac function. Ultrasound Obstet Gynecol 2007; 29 (3): 321-25.

33 Cruz-Martinez R, Figueras F, Bennasar M, Garcia-Posadas R, Crispi F, Hernandez-Andrade E, et al. Normal reference ranges from 11 to 41 weeks' gestation of fetal left modified myocardial performance index by conventional Doppler with the use of stringent criteria for delimitation of the time periods. Fetal Diagn Ther 2012; 32 (1-2): 79-86.

34 Eidem BW, Edwards JM, Cetta F. Quantitative assessment of fetal ventricular function: establishing normal values of the myocardial performance index in the fetus. Echocardiography 2001; 18 (1): 9-13.

35 Meriki N, Welsh AW. Development of Australian reference ranges for the left fetal modified myocardial performance index and the influence of caliper location on time interval measurement. Fetal Diagn Ther 2012; 32: 87-95.

36 Allan LD, Joseph MC, Boyd EG, Campbell S, Tynan M. M-mode echocardiography in the developing human fetus. Br Heart J 1982; 47 (6): 573-83.

37 Godfrey ME, Messing B, Valsky DV, Cohen SM, Yagel S. Fetal cardiac function: M-mode and 4D spatiotemporal image correlation. Fetal Diagn Ther 2012; 32 (1-2): 17-21.

38 DeVore GR, Siassi B, Platt LD. Fetal echocardiography. IV. M-mode assessment of ventricular size and contractility during the second and third trimesters of pregnancy in the normal fetus. Am J Obstet Gynecol 1984; 150 (8): 981-88.

39 Quinones MA, Pickering E, Alexander JK. Percentage of shortening of the echocardiographic left ventricular dimension. Its use in determining ejection fraction and stroke volume. Chest 1978; 74 (1): 59-65.

40 Carvalho JS, O'Sullivan C, Shinebourne EA, Henein MY. Right and left ventricular long-axis function in the fetus using angular M-mode. Ultrasound Obstet Gynecol 2001; 18 (6): 619-22.

41 Gardiner HM, Pasquini L, Wolfenden J, Barlow A, Li W, Kulinskaya $\mathrm{E}$, et al. Myocardial tissue Doppler and long axis function in the fetal heart. Int J Cardiol 2006; 113 (1): 39-47.

42 Cruz-Lemini M, Crispi F, Valenzuela-Alcaraz B, Figueras F, Sitges 
M, Gomez O, et al. Value of annular M-mode displacement versus tissue Doppler velocities to assess cardiac function in intrauterine growth restriction. Ultrasound Obstet Gynecol 2013; 42 (2): 175181.

43 Ho CY, Solomon SD. A clinician's guide to tissue Doppler imaging. Circulation 2006; 113 (10): e396-98.

44 Acharya G, Pavlovic M, Ewing L, Nollmann D, Leshko J, Huhta JC. Comparison between pulsed-wave Doppler- and tissue Dopplerderived Tei indices in fetuses with and without congenital heart disease. Ultrasound Obstet Gynecol 2008; 31 (4): 406-11.

45 Harada K, Tsuda A, Orino T, Tanaka T, Takada G. Tissue Doppler imaging in the normal fetus. Int J Cardiol 1999; 71 (3): 227-34.

46 Comas M, Crispi F, Cruz-Martinez R, Martinez JM, Figueras F, Gratacos E. Usefulness of myocardial tissue Doppler vs conventional echocardiography in the evaluation of cardiac dysfunction in earlyonset intrauterine growth restriction. Am J Obstet Gynecol 2010; 203 (1): 45. e1-7.

47 Yu CM, Sanderson JE, Marwick TH, Oh JK. Tissue Doppler imaging a new prognosticator for cardiovascular diseases. J Am Coll Cardiol 2007; 49 (19): 1903-14.

48 Crispi F, Sepulveda-Swatson E, Cruz-Lemini M, Rojas-Benavente J, Garcia-Posada R, Dominguez JM, et al. Feasibility and reproducibility of a standard protocol for $2 \mathrm{D}$ speckle tracking and tissue Doppler-based strain and strain rate analysis of the fetal heart. Fetal Diagn Ther 2012; 32 (1-2): 96-108.

49 Bijnens BH, Cikes M, Claus P, Sutherland GR. Velocity and deformation imaging for the assessment of myocardial dysfunction. Eur J Echocardiogr 2009; 10 (2): 216-26.

50 Van Mieghem T, Giusca S, DeKoninck P, Gucciardo L, Done E, Hindryckx A, et al. Prospective assessment of fetal cardiac function with speckle tracking in healthy fetuses and recipient fetuses of twin-to-twin transfusion syndrome. J Am Soc Echocardiogr; 23 (3): 301-8.

51 Yagel S, Cohen SM, Shapiro I, Valsky DV. 3D and 4D ultrasound in fetal cardiac scanning: a new look at the fetal heart. Ultrasound Obstet Gynecol 2007; 29 (1): 81-95.

52 Esh-Broder E, Ushakov FB. mbar T, Yagel S. Application of freehand three-dimensional echocardiography in the evaluation of fetal cardiac ejection fraction: a preliminary study. Ultrasound Obstet Gynecol 2004; 24: 546-5551.

53. Hamill N, Yeo L, Romero R, Hassan SS, Myers SA, Mittal P, et al. Fetal cardiac ventricular volume, cardiac output, and ejection fraction determined with 4-dimensional ultrasound using spatiotemporal image correlation and virtual organ computer-aided analysis. Am J Obstet Gynecol 2011; 205: 76. e1-e10.
54 Messing B, Cohen SM, Valsky DV, Rosenak D, Hochner-Celnikier $\mathrm{D}$, Savchev S, et al. Fetal cardiac ventricle volumetry in the second half of gestation assessed by 4D ultrasound using STIC combined with inversion mode. Ultrasound Obstet Gynecol 2007; 30 (2): 14251.

55 Hamill N, Romero R, Hassan SS, Lee W, Myers SA, Mittal P, et al. Repeatability and reproducibility of fetal cardiac ventricular volume calculations using spatiotemporal image correlation and virtual organ computer-aided analysis. J Ultrasound Med 2009; 28 (10): 1301-11.

56 Hamill N, Romero R, Hassan SS, Lee W, Myers SA, Mittal P, et al. Repeatability and reproducibility of fetal cardiac ventricular volume calculations using spatiotemporal image correlation and virtual organ computer-aided analysis. J Ultrasound Med 2009; 28 (10): 1301-11.

57 Friedman KG, Margossian R, Graham DA, Harrild DM, Emani SM, Wilkins-Haug LE, et al. Postnatal left ventricular diastolic function after fetal aortic valvuloplasty. Am J Cardiol; 108 (4): 556-60.

58 Cruz-Lemini M, Crispi F, Van Mieghem T, Pedraza D, CruzMartinez R, Acosta-Rojas R, et al. Risk of perinatal death in earlyonset intrauterine growth restriction according to gestational age and cardiovascular Doppler indices: a multicenter study. Fetal Diagn Ther 2012; 32 (1-2): 116-22.

59 McElhinney DB, Vogel M, Benson CB, Marshall AC, WilkinsHaug LE, Silva V, et al. Assessment of left ventricular endocardial fibroelastosis in fetuses with aortic stenosis and evolving hypoplastic left heart syndrome. Am J Cardiol 2010; 106 (12): 1792-7.

60 Pedra SR, Smallhorn JF, Ryan G, Chitayat D, Taylor GP, Khan R, et al. Fetal cardiomyopathies: pathogenic mechanisms, hemodynamic findings, and clinical outcome. Circulation 2002; 106 (5): 585-91.

61 Wieczorek A, Hernandez-Robles J, Ewing L, Leshko J, Luther S, Huhta J. Prediction of outcome of fetal congenital heart disease using a cardiovascular profile score. Ultrasound Obstet Gynecol 2008; 31 (3): 284-88.

62 Pereira S, Ganapathy R, Syngelaki A, Maiz N, Nicolaides KH. Contribution of fetal tricuspid regurgitation in first-trimester screening for major cardiac defects. Obstet Gynecol; 117 (6): 138491.

63 Cruz-Martinez R, Figueras F, Hernandez-Andrade E, Oros D, Gratacos E. Changes in myocardial performance index and aortic isthmus and ductus venosus Doppler in term, small-for-gestational age fetuses with normal umbilical artery pulsatility index. Ultrasound Obstet Gynecol; 38 (4): 400-5. 